\section{EXPERT OPINION}

1. Introduction

2. A brief overview of NOACs and their bleeding risks

3. Major bleeding management and reversal strategies

4. Direct antidotes to NOACs

5. Expert opinion

\title{
Antidotes to non-vitamin K oral anticoagulants: necessary or not?
}

\author{
Marco Proietti \& Gregory YH Lip ${ }^{\dagger}$ \\ ${ }^{\dagger}$ University of Birmingham, Centre for Cardiovascular Sciences, City Hospital, Birmingham, UK
}

In the last few years, a new category of anticoagulants have been developed, the non-vitamin K oral anticoagulants (NOACs). The NOACs are of two classes: the direct thrombin inhibitor, namely dabigatran etexilate; and the oral factor Xa inhibitors rivaroxaban, apixaban and edoxaban, which have been proven to be as effective and safe (and sometimes, superior) compared to warfarin in the treatment of both atrial fibrillation (AF) and venous thromboembolism (VTE). One major concern about their use has always been the lack of an effective antidote or reversal strategy. The objective of this editorial is to provide an overview of the characteristics of NOAC antidotes that are in development. Moreover, we review their likely place in the management of NOAC-related bleeding episodes.

Keywords: antidotes, bleeding risk, non-vitamin $\mathrm{K}$ oral anticoagulants, reversal strategies

Expert Opin. Pharmacother. [Early Online]

\section{Introduction}

Vitamin $\mathrm{K}$ antagonists (VKAs; i.e., warfarin) have always been the mainstay of the prevention of stroke and thromboembolism in atrial fibrillation (AF) [1]. However, the VKAs require monitoring and have important inter- and intra-patient variability leading to the requirement of regular International Normalized Ratio monitoring, to ensure a high percentage of time in therapeutic range. Indeed, even if a high time in therapeutic range is associated with the best efficacy and safety for the VKAs [1], the search for a much more manageable oral anticoagulant agent continued, due to the VKA-related bleeding risks (especially in patients with labile International Normalized Ratios) and the need for regular monitoring.

In the last few years, a new category of anticoagulants have been developed, the non-vitamin $\mathrm{K}$ oral anticoagulants (NOACs), formerly referred to as new or novel oral anticoagulants (Table 1) [2]. The NOACs are of two classes, the direct thrombin inhibitor, namely dabigatran etexilate; and the oral Factor Xa inhibitors, rivaroxaban, apixaban and edoxaban, which have been proved to be as effective and safe (and sometimes, superior) compared to warfarin in the treatment of both AF [3] and venous thromboembolism (VTE) [4].

However, when NOACs entered the current pharmacopeia, one major concern about their use has always been the lack of an effective antidote or reversal strategy [5]. With the recent announcement of the regulatory application submissions for the approval for use of a specific reversal agent to dabigatran etexilate, called idarucizumab [6], and the 'Orphan Drug' status designation for one Factor Xa inhibitors experimental antidote, andexanet alfa, the era of specific antidotes to NOACs seems to be increasingly more prominent.

\section{A brief overview of NOACs and their bleeding risks}

Dabigatran etexilate is a pro-drug with a potent thrombin direct inhibitor effect. After its oral administration, the drug is rapidly converted to its active form, reaching its peak plasma concentration within $2 \mathrm{~h}$ of its intake. Its half-life is around $15 \mathrm{~h}$ 
Table 1. NOACs general pharmacological aspects.

\begin{tabular}{lllll}
\hline & \multicolumn{1}{c}{ Rivaroxaban } & Apixaban & Edoxaban & Dabigatran \\
\hline Type of drug & Active agent & Active agent & Active agent & Inactive pro-drug \\
Action mechanism & Direct FXa inhibitor & Direct FXa inhibitor & Direct FXa inhibitor & Direct thrombin inhibitor \\
Time to peak effect (hours) & 2 & $3-4$ & $1-2$ & $1.5-2$ \\
Half-life (hours) & $5-9$ & $8-15$ & $9-10$ & $12-17$ \\
Oral bioavailability (\%) & 100 & 50 & 62 & 6 \\
Renal clearance (\%) & 33 & 25 & $35-39$ & 80 \\
\hline
\end{tabular}

NOACs: Non-vitamin K oral anticoagulants.

and the drug is almost completely excreted by the kidneys [5]. Rivaroxaban, apixaban and edoxaban are reversible inhibitors of Factor Xa, which are highly bioavailable [5], ranging from $50 \%$ of apixaban to almost $100 \%$ of rivaroxaban. The renal clearance of these NOACs is less marked compared to dabigatran, being approximately $25 \%$ with apixaban to around $50 \%$ with edoxaban [5].

The need for an available and effective antidote is clearly related to the occurrence of intracranial or other major lifethreatening bleeding episodes in a patient taking a NOAC. One of the main advantages in the adoption of NOACs instead of VKAs in daily clinical practice has been the lower incidence of bleeding episodes, especially intracranial hemorrhage (ICH) in the treatment of AF [7] or any major or clinically relevant bleeding in the treatment of VTE [4].

In the RE-LY study, for example, dabigatran demonstrated a reduced rate of $\mathrm{ICH}$ and life-threatening bleeding, both with 110 and $150 \mathrm{mg}$ twice a day doses, although gastrointestinal bleeding was higher with the $150 \mathrm{mg}$ dose [7]. Moreover, the outcome of ICH was perhaps better in the dabigatrantreated group, with an overall lower rate of fatal bleeding [8].

Of the Factor Xa inhibitors, rivaroxaban was associated with similar rates of major bleeding, but with a lower rate of ICH compared to warfarin [9]. Furthermore, both apixaban and edoxaban were associated with lower rates of major bleeding and $\mathrm{ICH}[10,11]$.

Finally, some recent evidence underlines how in certain clinical settings, as in hip replacement surgery or in medically ill patients, NOACs seem to bring a higher bleeding risk even if in these settings the use of NOACs is less common [12].

\section{Major bleeding management and reversal strategies}

As mentioned above, the major concern about NOACs has been the management of major bleeding with an effective reversal strategy $[5,7,13]$. The VKAs have a specific antidote, which is vitamin $\mathrm{K}$ - but even its administration requires $9-12 \mathrm{~h}$ to have an appreciable effect on reversing the coagulation abnormalities associated with VKA over-anticoagulation. In the interim, non-specific supportive measures are used, as described above.
Initial supportive measures, for example, immediately stop all antithrombotic drugs, mechanical hemostasis and hemodynamic support, are obviously recommended in all major bleeding episodes, including those occurring with a VKA or NOAC [5].

Oral charcoal may reduce dabigatran [13] and apixaban [14] absorption, if administered not too long after the last drug intake. In patients treated with dabigatran, the use of hemodialysis may remove the drug from the blood, thus reversing its anticoagulant effect [15]. Both prothrombin complex concentrate and activated prothrombin complex concentrate have shown various degrees of efficacy in restoring near-normality of various coagulation tests, and case series of their use in NOAC bleeding cases have been reported. Some efficacy has also been shown by the administration of recombinant FVIIIa [13]. A summary of effective reversal strategies is found in Table 2.

\section{Direct antidotes to NOACs}

In these last few years, the development of effective direct NOACs antidotes has made great advances (Table 2) [13]. Regarding such an important clinical problem, this issue has received a greater amount of attention recently [16].

Idarucizumab is a humanized antibody fragment that binds to dabigatran with an affinity approximately 350 times greater than thrombin, which has been recently submitted by Boehringer Ingelheim for regulatory approval as a specific antidote to dabigatran.

Idarucizumab binds dabigatran, preventing its bond to thrombin [17]. Thus, the dabigatran anticoagulant effect is almost immediately neutralized [17]. A recently published Phase II study [6] has shown its safety and tolerability in human subjects. Currently on-going is the REVERSE-AD study, that is, 'A Phase III Case Series Clinical Study of the Reversal of the Anticoagulant Effects of Dabigatran by Intravenous Administration of 5.0g Idarucizumab (BI 655075) in Patients Treated With Dabigatran Etexilate Who Have Uncontrolled Bleeding or Require Emergency Surgery or Procedures' (ClinicalTrials.gov Identifier: NCT02104947). This study was designed to test the efficacy of idarucizumab in patients undergoing major bleeding or emergency surgery 
Table 2. Reversal strategies and specific antidotes for NOACs [13].

\begin{tabular}{lll}
\hline & Non-specific reversal strategies & Targeted antidotes \\
\hline Dabigatran & PCC, Activated PCC, recombinant FVIla & Idarucizumab \\
Rivaroxaban & PCC, Activated PCC, recombinant FVIla & Andexanet Alfa, Ciraparantag \\
Apixaban & PCC, Activated PCC, recombinant FVIla & Andexanet Alfa, Ciraparantag \\
Edoxaban & PCC, Activated PCC, recombinant FVlla & Andexanet Alfa, Ciraparantag \\
\hline
\end{tabular}

PCC: Prothrombin complex concentrate; NOACs: Non-vitamin K oral anticoagulants.

or procedures. The main outcome is the maximum reversal of anticoagulant activity after a 4-h long intravenous fusion.

For the Factor Xa inhibitors, another experimental antidote, andexanet alfa, has also been submitted for regulatory approval. Even in this case, it is an engineered molecule, obtained by manipulating and inactivating Factor Xa from a pro-coagulant point of view, but being still able to bind the inhibitors. Indeed, andexanet alfa has been shown to immediately reverse the anticoagulant effect of Factor $\mathrm{Xa}$ inhibitors [18]. For example, preliminary data from a phase III study about the treatment of human subjects taking apixaban anticoagulant therapy (NCT02207725) show how a rapid $400 \mathrm{mg}$ andexanet alfa intravenous administration results in a complete and rapid normalization of anti-factor Xa activity levels [19]. Moreover, recent results from the ANNEXA-A study showed that a bolus infusion of andexanet alfa, a rapid normalization of anti-factor $\mathrm{Xa}$ and thrombin levels could be achieved [20].

Another similar study, examining the effect of andexanet alfa on rivaroxaban activity (NCT02220725) is currently on-going, whereas a third study on the efficacy of andexanet alfa on all subjects taking Factor Xa inhibitors is still recruiting patients (NCT02329327).

Finally, as another possible option for the reversal of NOACs, the small molecule aripazine, now known as ciraparantag, seems to be promising, being able to inhibit dabigatran and the Factor Xa inhibitors, but for now specific data about this new agent are limited [19].

\section{Expert opinion}

NOACs have now entered in our daily routine practice, but the concerns of bleeding and how to treat major bleeding episodes concern physicians who would otherwise willingly prescribe these drugs, given their proved efficacy. All the NOACs have half-life times that would permit loss of their anticoagulant effect in a relatively rapid time, and the initial supportive management strategy seems to be appropriate.

Furthermore, the use of NOACs seems to be relatively safer than warfarin, with significantly lower rates of serious bleeding (especially, intracranial bleeding), reducing the fear of physicians in prescribing these drugs, notwithstanding the lack of a specific antidote until recently.

Beyond all that, the development of new, highly engineered molecules able to specifically block and rapidly reverse NOACs has helped to facilitate bleeding management.

In life-threatening situations, the possibility to barely immediately reverse the anticoagulation effect of NOACs could have importance. For example, when hemodynamic stability has been compromised during hemorrhagic shock, stopping active bleeding could be necessary in trying to stabilize vital functions.

Whilst the development of such antidotes may allow greater dissemination of NOACs, additional large-scale studies would be needed to prove the effectiveness of these molecules. First of all, sample size for these studies should be tailored according to clinical end points and not just on pharmacokinetics end points. Second, the power of the studies should be adequate to show superiority. Another aspect is the absence of clinically relevant side effects and cost-effectiveness.

Major bleeding can be related to high health-related costs. Also the costs in terms of quality of life in patients with major bleeding can be substantial.

\section{Declaration of interest}

GYH Lip has served as an advisor or consultant for: Astellas Pharma, Inc.; Bayer HealthCare Pharamceuticals; BIOTRONIK; Boehringer Ingelheim Pharmaceuticals, Inc.; BristolMyers Squibb Company; Daiichi Sankyo, Inc.; Merck \& Co., Inc.; Pfizer, Inc.; Portola Pharmaceuticals, Inc. and Sanofi. GYH Lip has also served as a speaker of a member of a speakers bureau for: Bayer HealthCare Pharmaceuticals; Boehringer Ingelheim Pharmaceuticals, Inc.; Bristol-Myers Squibb Company; Pfizer Inc. and Sanofi. The authors have no other relevant affiliations or financial involvement with any organization or entity with a financial interest in or financial conflict with the subject matter or materials discussed in the manuscript apart from those disclosed. 


\section{Bibliography}

Papers of special note have been highlighted as either of interest $(\bullet)$ or of considerable interest

$(\bullet)$ to readers.

1. De Caterina R, Husted S, Wallentin L, et al. Vitamin $\mathrm{K}$ antagonists in heart disease: Current status and perspectives (Section III). Thromb Haemost 2013;110(6):1087-107

- $\quad$ Establishes the efficacy of warfarin therapy in preventing adverse outcomes in atrial fibrillation (AF).

2. Husted S, de Caterina R, Andreotti F, et al. Non-vitamin $\mathrm{K}$ antagonist oral anticoagulants (NOACs): No longer new or novel. Thromb Haemost 2014;111(5):781-2

- $\quad$ ESC Expert Opinion on the nomenclature of non-Vitamin $\mathrm{K}$ oral anticoagulants (NOACs).

3. Skjøth F, Larsen TB, Rasmussen LH, Lip GYH. Efficacy and safety of edoxaban in comparison with dabigatran, rivaroxaban and apixaban for stroke prevention in atrial fibrillation. An indirect comparison analysis. Thromb Haemost 2014;111(5):981-8

- $\quad$ Establishes the efficacy and safety of NOACs in AF.

4. Hirschl M, Kundi M. New oral anticoagulants in the treatment of acute venous thromboembolism - a systematic review with indirect comparisons. Vasa 2014;43(5):353-64

-• Establishes the efficacy and safety of NOACs in venous thromboembolism (VTE).

5. Schulman S. New oral anticoagulant agents - general features and outcomes in subsets of patients. Thromb Haemost 2014;111(4):575-82

- Defines characteristics of NOACs.

6. Glund S, Moschetti V, Norris S, et al. A randomised study in healthy volunteers to investigate the safety, tolerability and pharmacokinetics of idarucizumab, a specific antidote to dabigatran.

Thromb Haemost 2015;113(5):943-51

-. Safety and tolerability of idarucizumab.

7. Chan NC, Paikin JS, Hirsh J, et al. New oral anticoagulants for stroke prevention in atrial fibrillation: Impact of study design, double counting and unexpected findings on interpretation of study results and conclusions. Thromb Haemost

2014;111:798-807

- Refines characteristics of NOACs.

8. Hart RG, Diener H-C, Yang S, et al. Intracranial hemorrhage in atrial fibrillation patients during anticoagulation with warfarin or dabigatran: the RE-LY trial. Stroke 2012;43(6):1511-17

•• Safety of dabigatran on intracranial hemorrhage (ICH).

9. Patel MR, Mahaffey KW, Garg J, et al. Rivaroxaban versus warfarin in nonvalvular atrial fibrillation. N Engl J Med 2011;365(10):883-91

- Safety of dabigatran on ICH.

10. Hylek EM, Held C, Alexander JH, et al. Major bleeding in patients with atrial fibrillation receiving apixaban or warfarin: The ARISTOTLE trial (Apixaban for reduction in stroke and other thromboembolic events in atrial fibrillation): Predictors, characteristics, and clinical outcomes. J Am Coll Cardiol 2014;63:2141-7

- Safety of rivaroxaban on ICH.

11. Giugliano RP, Ruff CT, Braunwald E, et al. Edoxaban versus warfarin in patients with atrial fibrillation. N Engl J Med 2013;369:2093-104

- Safety of edoxaban on ICH.

12. Sardar P, Chatterjee S, Lavie CJ, et al. Risk of major bleeding in different indications for new oral anticoagulants: insights from a meta-analysis of approved dosages from 50 randomized trials. Int J Cardiol 2015;179:279-87

- Refines the analysis for major bleeding risk of NOACs.

13. Greinacher A, Thiele T, Selleng K. Reversal of anticoagulants: an overview of current developments. Thromb Haemost 2015;113(5):931-42

-. Establishes characteristics of NOAC reversal strategies and antidotes.

14. Wang X, Mondal S, Wang J, et al. Effect of activated charcoal on apixaban pharmacokinetics in healthy subjects. Am J Cardiovasc Drugs 2014;14(2):147-54

- Refines the analysis of reversal strategies for apixaban.
15. Khadzhynov D, Wagner F, Formella S, et al. Effective elimination of dabigatran by haemodialysis. A phase I single-centre study in patients with end-stage renal disease. Thromb Haemost 2013;109(4):596-605

- Refines the analysis of reversal strategies for dabigatran.

16. Gomez-Outes A, Suarez-Gea ML, Lecumberri R, et al. Specific antidotes in development for reversal of novel anticoagulants: a review. Recent Pat Cardiovasc Drug Discov 2014;9(1):2-10

- Refines the analysis of NOAC reversal strategies and antidotes.

17. Schiele F, van Ryn J, Canada K, et al. A specific antidote for dabigatran: functional and structural characterization. Blood 2013;121(18):3554-62

-• Describes characteristics of dabigatran antidote.

18. Lu G, DeGuzman FR, Hollenbach SJ, et al. A specific antidote for reversal of anticoagulation by direct and indirect inhibitors of coagulation factor Xa. Nat Med 2013;19(4):446-51

-• Describes characteristics of factor Xa inhibitors antidote.

19. Siegal DM. Managing target-specific oral anticoagulant associated bleeding including an update on pharmacological reversal agents. J Thromb Thrombolysis 2015;39(3):395-402

- Refines the analysis of NOAC reversal strategies and antidotes.

20. Late-Breaking Clinical Trial Abstracts. Circulation 2014;130(23):2105-26

-. Describes characteristics of factor $\mathrm{Xa}$ inhibitors antidote.

Affiliation

Marco Proietti ${ }^{1,2} \&$ Gregory YH Lip ${ }^{\dagger 1,3}$

${ }^{\dagger}$ Author for correspondence

${ }^{1}$ University of Birmingham, Centre for

Cardiovascular Sciences, City Hospital,

Birmingham, UK

${ }^{2}$ I Clinica Medica, Department of Internal

Medicine and Medical Specialties, Sapienza-

University of Rome, Rome, Italy

${ }^{3}$ Aalborg Thrombosis Research Unit, Department of Clinical Medicine, Aalborg University,

Aalborg, Denmark

Tel: +44 121507 5080;

E-mail: g.y.h.lip@bham.ac.uk 